Research Article

\title{
New Oscillatory Behavior of Second-Order Nonlinear Dynamic Equations with Damping on Time Scales
}

\author{
Shouhua Liu and Quanxin Zhang \\ Department of Mathematics, Binzhou University, Shandong 256603, China \\ Correspondence should be addressed to Quanxin Zhang; 3314744@163.com \\ Received 21 April 2014; Revised 28 May 2014; Accepted 29 May 2014; Published 9 June 2014 \\ Academic Editor: Tongxing Li \\ Copyright (C) 2014 S. Liu and Q. Zhang. This is an open access article distributed under the Creative Commons Attribution License, \\ which permits unrestricted use, distribution, and reproduction in any medium, provided the original work is properly cited. \\ We establish four new oscillation criteria of Grace-type for the second-order nonlinear dynamic equations with damping. These \\ criteria extend known criteria for corresponding dynamic equations. Our results are new even in the continuous and the discrete \\ cases.
}

\section{Introduction}

In this paper, we deal with the oscillatory behavior of all solutions of the second-order nonlinear dynamic equation with damping:

$$
\begin{gathered}
\left(a(t)\left(x^{\Delta}(t)\right)^{\gamma}\right)^{\Delta}+p(t)\left(x^{\Delta}(t)\right)^{\gamma} \\
+q(t) f(x(t))=0, \\
t \in \mathbb{T}, \quad t \geq t_{0} .
\end{gathered}
$$

Our aim is to give some oscillatory criteria of Grace-type of (1). Here, we give the following hypotheses:

$\left(\mathrm{H}_{1}\right) \mathbb{T}$ is a time scale (i.e., a nonempty closed subset of the real numbers $\mathbb{R}$ ) which is unbounded above and $t_{0} \epsilon$ $\mathbb{T}$ with $t_{0}>0$; we define the time scale interval of the form $\left[t_{0}, \infty\right)_{\mathbb{T}}$ by $\left[t_{0}, \infty\right)_{\mathbb{T}}=\left[t_{0}, \infty\right) \cap \mathbb{T}$;

$\left(\mathrm{H}_{2}\right) \quad \gamma \geq 1$ is the ratio of two positive and odd integers;

$\left(\mathrm{H}_{3}\right) \quad a, p, q: \mathbb{T} \rightarrow \mathbb{R}$ are positive and rd-continuous functions such that $-p / a \in \mathscr{R}^{+}$;

$\left(\mathrm{H}_{4}\right) f: \mathbb{R} \rightarrow \mathbb{R}$ is a continuous function such that, for some positive constant $L$,

$$
\frac{f(x)}{x^{\gamma}} \geq L \quad \forall x \neq 0 .
$$

By a solution of (1), we mean a nontrivial real-valued function $x$ satisfying (1) for $t \in \mathbb{T}$. We recall that a solution $x$ of (1) is said to be oscillatory on $\left[t_{0}, \infty\right)_{\mathbb{T}}$ in case it is neither eventually positive nor eventually negative; otherwise, the solution is said to be nonoscillatory. Equation (1) is said to be oscillatory in case all of its solutions are oscillatory. Our attention is restricted on those solutions $x$ of (1) in which $x$ is not the eventually identical zero.

In 2009, Grace et al. [1] considered the second-order halflinear dynamic equations:

$$
\begin{gathered}
\left(a(t)\left(x^{\Delta}(t)\right)^{\gamma}\right)^{\Delta}+q(t) x^{\gamma}(t)=0, \\
t \in \mathbb{T}, \quad t \geq t_{0},
\end{gathered}
$$

and obtained some oscillatory criteria of Grace-type of (3). In recent years, there have been numerous researches and many research activities concerning the oscillation and nonoscillation of solutions of (3) and its special cases; we refer the reader to the papers [2-8].

In this paper, we will establish two sufficient conditions for oscillation of all solutions of (1) by use of the generalized Riccati transformation and the inequality technique, under the condition that

$$
\int_{t_{0}}^{\infty}\left[\frac{1}{a(t)} e_{-p / a}\left(t, t_{0}\right)\right]^{1 / \gamma} \Delta t=\infty .
$$

Moreover, if condition (4) does not hold, that is,

$$
\int_{t_{0}}^{\infty}\left[\frac{1}{a(t)} e_{-p / a}\left(t, t_{0}\right)\right]^{1 / \gamma} \Delta t<\infty
$$


holds, two sufficient conditions are obtained for oscillation or convergence to zero of (1).

In order to prove the main results of this paper, we will use the following rules:

$$
\begin{gathered}
(f g)^{\Delta}(t)=f^{\Delta}(t) g(t)+f(\sigma(t)) g^{\Delta}(t) \\
=f(t) g^{\Delta}(t)+f^{\Delta}(t) g(\sigma(t)), \\
\left(\frac{f}{g}\right)^{\Delta}(t)=\frac{f^{\Delta}(t) g(t)-f(t) g^{\Delta}(t)}{g(t) g(\sigma(t))} \quad \text { if } g g^{\sigma} \neq 0 .
\end{gathered}
$$

For more details about differential and integral theory on the time scale, see $[9,10]$.

\section{The Main Results}

In order to prove the main results of this paper, we first give the following two lemmas.

Lemma 1 (see [9, Theorem 1.90]). Assume that $x$ is deltadifferentiable and eventually positive or eventually negative; then

$$
\left((x(t))^{\gamma}\right)^{\Delta}=\gamma \int_{0}^{1}[h x(\sigma(t))+(1-h) x(t)]^{\gamma-1} x^{\Delta}(t) \mathrm{d} h .
$$

Lemma 2. Assume that $\left(H_{1}\right),\left(H_{2}\right)$, and (4) hold. Let $x(t)$ be an eventually position solution of (1). Then there exists $t_{1} \in$ $\left[t_{0}, \infty\right)_{\mathbb{V}}$ such that

$$
x^{\Delta}(t)>0, \quad\left[a(t)\left(x^{\Delta}(t)\right)^{\gamma}\right]^{\Delta}<0 \quad \forall t \in\left[t_{1}, \infty\right)_{\mathbb{T}} .
$$

Similar to the proof of Lemma 3.3 in paper [8], we can give the proof of Lemma 2; thus, the proof is omitted here.

Theorem 3. Assume that $\left(H_{1}\right)-\left(H_{4}\right)$ and (4) hold. If there exists a positive nondecreasing $\Delta$-differentiable function $\delta \in$ $C_{r d}^{1}\left(\left[t_{0}, \infty\right)_{\mathbb{T}}, \mathbb{R}\right)$ such that, for every $t_{1} \in\left[t_{0}, \infty\right)_{\mathbb{T}}$,

$$
\begin{aligned}
\limsup _{t \rightarrow \infty} \int_{t_{1}}^{t} & {\left[L \delta(s) q(s)-\eta^{\gamma}(s)\left(\delta^{\Delta}(s)-\delta(s) \frac{p(s)}{a(s)}\right)\right] } \\
& \times \Delta s=\infty,
\end{aligned}
$$

where

$$
\eta(t)=\left(\int_{t_{1}}^{t}\left(\frac{1}{a(s)}\right)^{1 / \gamma} \Delta s\right)^{-1}
$$

then $(1)$ is oscillatory on $\left[t_{0}, \infty\right)_{\mathbb{T}}$.

Proof. Suppose, to the contrary, that $x(t)$ is a nonoscillatory solutions of $(1)$ on $\left[t_{0}, \infty\right)_{\mathbb{T}}$. Without loss of generality, we may assume that $x(t)>0$ for all $t \in\left[t_{1}, \infty\right)_{\mathbb{V}}, t_{1} \in\left[t_{0}, \infty\right)_{\mathbb{V}}$. We shall consider only this case, since the proof when $x(t)$ is eventually negative is similar. By Lemma 2, we obtain on $\left[t_{1}, \infty\right)_{\mathbb{W}}$ that

$$
\begin{gathered}
\frac{1}{(x(t))^{\gamma}} \geq \frac{1}{(x(\sigma(t)))^{\gamma}}, \\
a(t)\left(x^{\Delta}(t)\right)^{\gamma} \geq a(\sigma(t))\left(x^{\Delta}(\sigma(t))\right)^{\gamma},
\end{gathered}
$$

Define the function $W(t)$ by

$$
W(t)=\delta(t) \frac{a(t)\left(x^{\Delta}(t)\right)^{\gamma}}{(x(t))^{\gamma}}, \quad t \in\left[t_{1}, \infty\right)_{\mathbb{T}} .
$$

Then, we have $W(t)>0$ on $\left[t_{1}, \infty\right)_{\mathbb{T}}$; by (1), (6), (7), and (12), we obtain

$$
\begin{aligned}
& W^{\Delta}(t)=\frac{\delta(t)}{(x(t))^{\gamma}}\left(a(t)\left(x^{\Delta}(t)\right)^{\gamma}\right)^{\Delta} \\
& +a(\sigma(t))\left(x^{\Delta}(\sigma(t))\right)^{\gamma} \\
& \times \frac{(x(t))^{\gamma} \delta^{\Delta}(t)-\delta(t)\left((x(t))^{\gamma}\right)^{\Delta}}{(x(t))^{\gamma}(x(\sigma(t)))^{\gamma}} \\
& \leq-\operatorname{Lq}(t) \delta(t)-\frac{\delta(t) p(t)\left(x^{\Delta}(t)\right)^{\gamma}}{(x(t))^{\gamma}} \\
& +\frac{a(\sigma(t))\left(x^{\Delta}(\sigma(t))\right)^{\gamma} \delta^{\Delta}(t)}{(x(\sigma(t)))^{\gamma}} \\
& -\frac{\delta(t) a(\sigma(t))\left(x^{\Delta}(\sigma(t))\right)^{\gamma}\left((x(t))^{\gamma}\right)^{\Delta}}{(x(t))^{\gamma}(x(\sigma(t)))^{\gamma}} \\
& \leq-L q(t) \delta(t)-\frac{\delta(t) p(t)\left(x^{\Delta}(t)\right)^{\gamma}}{(x(t))^{\gamma}} \\
& +\frac{a(\sigma(t))\left(x^{\Delta}(\sigma(t))\right)^{\gamma} \delta^{\Delta}(t)}{(x(\sigma(t)))^{\gamma}} \\
& \leq-L q(t) \delta(t)-\delta(t) \frac{p(t)}{a(t)} \frac{a(t)\left(x^{\Delta}(t)\right)^{\gamma}}{(x(t))^{\gamma}} \\
& +\delta^{\Delta}(t) \frac{a(t)\left(x^{\Delta}(t)\right)^{\gamma}}{(x(t))^{\gamma}} \\
& =-L q(t) \delta(t) \\
& +\left(\delta^{\Delta}(t)-\delta(t) \frac{p(t)}{a(t)}\right) a(t)\left(\frac{x^{\Delta}(t)}{x(t)}\right)^{\gamma} .
\end{aligned}
$$

Now

$$
\begin{aligned}
x(t) & =x\left(t_{1}\right)+\int_{t_{1}}^{t} x^{\Delta}(s) \Delta s \\
& =x\left(t_{1}\right)+\int_{t_{1}}^{t}\left(\frac{1}{a(s)}\right)^{1 / \gamma}(a(s))^{1 / \gamma} x^{\Delta}(s) \Delta s \\
& \geq\left(\int_{t_{1}}^{t}\left(\frac{1}{a(s)}\right)^{1 / \gamma} \Delta s\right)\left(a(t)\left(x^{\Delta}(t)\right)^{\gamma}\right)^{1 / \gamma},
\end{aligned}
$$

and thus

$$
\begin{aligned}
\left(\frac{x^{\Delta}(t)}{x(t)}\right)^{\gamma} & \leq \frac{1}{a(t)}\left(\int_{t_{1}}^{t}\left(\frac{1}{a(s)}\right)^{1 / \gamma} \Delta s\right)^{-\gamma} \\
& =\frac{\eta^{\gamma}(t)}{a(t)} \quad \text { for } t \in\left[t_{1}, \infty\right)_{\mathbb{T}} .
\end{aligned}
$$


Using (17) in (15), we have

$$
\begin{aligned}
W^{\Delta}(t) \leq & -L q(t) \delta(t) \\
& +\eta^{\gamma}(t)\left[\delta^{\Delta}(t)-\delta(t) \frac{p(t)}{a(t)}\right] \\
& \text { on }\left[t_{1}, \infty\right)_{\mathbb{T}} .
\end{aligned}
$$

Integrating (18) from $t_{1}$ to $t$, we obtain

$$
\begin{aligned}
0< & W(t) \leq W\left(t_{1}\right) \\
& -\int_{t_{1}}^{t}\left[L \delta(s) q(s)-\eta^{\gamma}(s)\left(\delta^{\Delta}(s)-\delta(s) \frac{p(s)}{a(s)}\right)\right] \Delta s,
\end{aligned}
$$

which gives a contradiction by (10). This completes the proof.

Theorem 4. Assume that $\left(\mathrm{H}_{1}\right)-\left(\mathrm{H}_{4}\right)$ and (4) hold. If there exists a positive $\Delta$-differentiable function $\delta \in C_{r d}^{1}\left(\left[t_{0}, \infty\right)_{\mathbb{T}}, \mathbb{R}\right)$ such that, for every $t_{1} \in\left[t_{0}, \infty\right)_{\mathbb{T}}$,

$$
\begin{aligned}
\limsup _{t \rightarrow \infty} \int_{t_{1}}^{t}\left[L q(s) \delta(s)-\frac{(a(s))^{1 / \gamma}(\eta(\sigma(s)))^{\gamma-1}}{4 \gamma \delta(s)}\right. \\
\left.\quad \times\left(\delta^{\Delta}(s)-\delta(s) \frac{p(s)}{a(s)}\right)^{2}\right] \Delta s=\infty,
\end{aligned}
$$

where $\eta$ has been defined in (11), then (1) is oscillatory on $\left[t_{0}, \infty\right)_{\mathbb{T}}$.

Proof. Suppose, to the contrary, that $x(t)$ is a nonoscillatory solution of $(1)$ on $\left[t_{0}, \infty\right)_{\mathbb{T}}$. We may assume, without loss of generality, that $x(t)>0$ for all $t \in\left[t_{1}, \infty\right)_{\mathbb{T}}, t_{1} \in$ $\left[t_{0}, \infty\right)_{\mathbb{T}}$. When $x(t)$ is eventually negative, the proof is similar. Proceeding as in the proof of Theorem 3, we obtain (14). Using (8) and (9), we have

$$
\begin{aligned}
\left((x(t))^{\gamma}\right)^{\Delta} & =\gamma \int_{0}^{1}[h x(\sigma(t))+(1-h) x(t)]^{\gamma-1} x^{\Delta}(t) \mathrm{d} h \\
& \geq \gamma \int_{0}^{1}[h x(t)+(1-h) x(t)]^{\gamma-1} x^{\Delta}(t) \mathrm{d} h \\
& =\gamma(x(t))^{\gamma-1}(x(t))^{\Delta}>0 .
\end{aligned}
$$

Using (12) and (21) in (14), we have

$$
\begin{aligned}
W^{\Delta}(t) \leq & -L q(t) \delta(t)-\frac{\delta(t)}{\delta(\sigma(t))} \frac{p(t)}{a(t)} W(\sigma(t)) \\
& +\frac{\delta^{\Delta}(t)}{\delta(\sigma(t))} W(\sigma(t)) \\
& -\frac{\gamma \delta(t) a(\sigma(t))\left(x^{\Delta}(\sigma(t))\right)^{\gamma} x^{\Delta}(t)}{(x(\sigma(t)))^{\gamma+1}}
\end{aligned}
$$

$$
\begin{aligned}
= & -\operatorname{Lq}(t) \delta(t) \\
& +\left[\frac{\delta^{\Delta}(t)}{\delta(\sigma(t))}-\frac{\delta(t)}{\delta(\sigma(t))} \frac{p(t)}{a(t)}\right] W(\sigma(t)) \\
& -\frac{\gamma \delta(t) a(\sigma(t))\left(x^{\Delta}(\sigma(t))\right)^{\gamma} x^{\Delta}(t)}{(x(\sigma(t)))^{\gamma+1}} .
\end{aligned}
$$

By $\left(a(t)\left(x^{\Delta}(t)\right)^{\gamma}\right)^{\Delta}<0$, we have

$$
\begin{aligned}
& a(t)\left(x^{\Delta}(t)\right)^{\gamma} \geq a(\sigma(t))\left(x^{\Delta}(\sigma(t))\right)^{\gamma} \\
& \text { i.e. } x^{\Delta}(t) \geq \frac{(a(\sigma(t)))^{1 / \gamma}}{(a(t))^{1 / \gamma}} x^{\Delta}(\sigma(t)) .
\end{aligned}
$$

Substituting (23) in (22), we obtain

$$
\begin{aligned}
W^{\Delta}(t) \leq & -L q(t) \delta(t) \\
& +\left[\frac{\delta^{\Delta}(t)}{\delta(\sigma(t))}-\frac{\delta(t)}{\delta(\sigma(t))} \frac{p(t)}{a(t)}\right] W(\sigma(t)) \\
& -\frac{\gamma \delta(t)}{(a(t))^{1 / \gamma}(\delta(\sigma(t)))^{(\gamma+1) / \gamma}} \\
& \times(W(\sigma(t)))^{(\gamma+1) / \gamma} \text { on }\left[t_{1}, \infty\right)_{\mathbb{T}} .
\end{aligned}
$$

That is, on $\left[t_{1}, \infty\right)_{\mathbb{T}}$,

$$
\begin{aligned}
W^{\Delta}(t) \leq & -L q(t) \delta(t) \\
& +\left[\frac{\delta^{\Delta}(t)}{\delta(\sigma(t))}-\frac{\delta(t)}{\delta(\sigma(t))} \frac{p(t)}{a(t)}\right] W(\sigma(t)) \\
& -\frac{\gamma \delta(t)}{(a(t))^{1 / \gamma}(\delta(\sigma(t)))^{(\gamma+1) / \gamma}} \\
& \times(W(\sigma(t)))^{(1-\gamma) / \gamma}(W(\sigma(t)))^{2} .
\end{aligned}
$$

Now using inequality (17) as the form, that is,

$$
\frac{x(t)}{x^{\Delta}(t)} \geq \frac{a^{1 / \gamma}(t)}{\eta(t)},
$$

this implies on $\left[t_{1}, \infty\right)_{\mathbb{T}}$ that

$$
\begin{aligned}
(W(t))^{(1-\gamma) / \gamma} & =(\delta(t) a(t))^{(1-\gamma) / \gamma}\left(\frac{x(t)}{x^{\Delta}(t)}\right)^{\gamma-1} \\
& \geq(\delta(t) a(t))^{(1-\gamma) / \gamma} \frac{a^{(1-\gamma) / \gamma}(t)}{\eta^{\gamma-1}(t)} \\
& =\delta^{(1-\gamma) / \gamma}(t) \eta^{1-\gamma}(t) .
\end{aligned}
$$


Using (27) in (25), we have on $\left[t_{1}, \infty\right)_{\mathbb{T}}$ that

$$
\begin{aligned}
& W^{\Delta}(t) \leq-L q(t) \delta(t) \\
& +\left[\frac{\delta^{\Delta}(t)}{\delta(\sigma(t))}-\frac{\delta(t)}{\delta(\sigma(t))} \frac{p(t)}{a(t)}\right] W(\sigma(t)) \\
& -\frac{\gamma \delta(t)}{(a(t))^{1 / \gamma}(\delta(\sigma(t)))^{2}} \\
& \times(\eta(\sigma(t)))^{1-\gamma}(W(\sigma(t)))^{2} \\
& =-L q(t) \delta(t)+\frac{(a(t))^{1 / \gamma}(\eta(\sigma(t)))^{\gamma-1}}{4 \gamma \delta(t)} \\
& \times\left(\delta^{\Delta}(t)-\delta(t) \frac{p(t)}{a(t)}\right)^{2} \\
& -\left[\frac{\sqrt{\gamma \delta(t)(\eta(\sigma(t)))^{1-\gamma}}}{\delta(\sigma(t)) \sqrt{(a(t))^{1 / \gamma}}} W(\sigma(t))\right. \\
& -\frac{\sqrt{(a(t))^{1 / \gamma}}}{2 \sqrt{\gamma \delta(t)(\eta(\sigma(t)))^{1-\gamma}}} \\
& \left.\times\left(\delta^{\Delta}(t)-\delta(t) \frac{p(t)}{a(t)}\right)\right]^{2} \\
& \leq-\left[L q(t) \delta(t)-\frac{(a(t))^{1 / \gamma}(\eta(\sigma(t)))^{\gamma-1}}{4 \gamma \delta(t)}\right. \\
& \left.\times\left(\delta^{\Delta}(t)-\delta(t) \frac{p(t)}{a(t)}\right)^{2}\right] .
\end{aligned}
$$

Integrating both sides of this inequality from $t_{1}$ to $t$, taking the lim sup of the resulting inequality as $t \rightarrow \infty$ and applying condition (20), we obtain a contradiction to the fact that $W(t)>0$ for $t \in\left[t_{1}, \infty\right)_{\mathbb{T}}$. This completes the proof.

Now, when condition (5) holds, using the same method of proof of Theorem 3.3 and Theorem 3.4 in paper [8], we can obtain the following theorems.

Theorem 5. Assume that $\left(H_{1}\right)-\left(H_{4}\right)$ and (5) hold. If there exists a positive $\Delta$-differentiable function $\delta \in C_{r d}^{1}\left(\left[t_{0}, \infty\right)_{\mathbb{T}}, \mathbb{R}\right)$ such that (10) holds, where $\eta$ has been defined in (11), and

$$
\int_{t_{0}}^{\infty}\left(\frac{1}{a(t)} \int_{t_{0}}^{t} e_{-p / a}(t, \sigma(s)) q(s) \Delta s\right)^{1 / \gamma} \Delta t=\infty,
$$

then every solution of (1) is either oscillatory or converges to zero on $\left[t_{0}, \infty\right)_{\mathbb{T}}$.

Theorem 6. Assume that $\left(H_{1}\right)-\left(H_{4}\right),(5)$, and (29) hold. If there exists a positive $\Delta$-differentiable function $\delta \in$ $C_{r d}^{1}\left(\left[t_{0}, \infty\right)_{\mathbb{T}}, \mathbb{R}\right)$ such that $(20)$ holds, where $\eta$ has been defined in (11), then every solution of (1) is either oscillatory or converges to zero on $\left[t_{0}, \infty\right)_{\mathbb{T}}$.

Remark 7. Our results in this paper extend some known results and make some results of [1] special cases of our results. The theorems in this paper are new even for the cases $\mathbb{T}=\mathbb{R}$ and $\mathbb{T}=\mathbb{Z}$.

Example 8. Consider the second-order nonlinear delay 2difference equations:

$$
\begin{gathered}
\left(t^{2 / 3}\left(x^{\Delta}(t)\right)^{5 / 3}\right)^{\Delta}+\frac{1}{t^{2}}\left(x^{\Delta}(t)\right)^{5 / 3} \\
+t^{-8 / 5}(x(t))^{5 / 3}\left(1+x^{2}(t)\right)=0 \\
t \in \overline{2^{\mathbb{Z}}}, \quad t \geq t_{0}:=2 .
\end{gathered}
$$

Here,

$$
\begin{gathered}
a(t)=t^{2 / 3}, \quad p(t)=\frac{1}{t^{2}}, \\
q(t)=t^{-8 / 5}, \quad f(x)=x^{5 / 3}\left(1+x^{2}\right), \quad \gamma=\frac{5}{3} .
\end{gathered}
$$

The conditions $\left(\mathrm{H}_{1}\right)$ and $\left(\mathrm{H}_{2}\right)$ are clearly satisfied, $\left(\mathrm{H}_{4}\right)$ holds with $L=1$, and $\left(\mathrm{H}_{3}\right)$ is satisfied as

$$
1-\mu(t) \frac{p(t)}{a(t)}=1-t \cdot \frac{1}{t^{8 / 3}}=1-\frac{1}{t^{5 / 3}}>0 \quad \text { for } t \geq 2 .
$$

Next, by [11, Lemma 2], using $\left(\mathrm{H}_{3}\right)$, we have

$$
\begin{aligned}
e_{-p / a}(t, 2) & \geq 1-\int_{2}^{t} \frac{p(s)}{a(s)} \Delta s \\
& =1-\int_{2}^{t} s^{-8 / 3} \Delta s=1-\frac{t^{-5 / 3}-2^{-5 / 3}}{2^{-5 / 3}-1} \\
& =\frac{t^{-5 / 3}+1-2^{-2 / 3}}{1-2^{-5 / 3}} \\
& \geq t^{-5 / 3}+1-2^{-2 / 3}>1-2^{-2 / 3}>\frac{1}{3}
\end{aligned}
$$

for $t \geq 2$, so that, as $t \rightarrow \infty$,

$$
\begin{aligned}
\int_{2}^{t}[ & \left.\frac{1}{a(s)} e_{-p / a}(s, 2)\right]^{1 / \gamma} \Delta s \\
\quad & \int_{2}^{t}\left[s^{-2 / 3} e_{-p / a}(s, 2)\right]^{3 / 5} \Delta s \\
& \geq \frac{1}{3^{3 / 5}} \int_{2}^{t} s^{-2 / 5} \Delta s=\frac{1}{3^{3 / 5}} \frac{t^{3 / 5}-2^{3 / 5}}{2^{3 / 5}-1} \longrightarrow \infty .
\end{aligned}
$$

Hence, (4) is satisfied. Now let $\delta(t)=t^{3 / 5}$; for all $t>s \geq 2$,

$$
\begin{aligned}
\eta(t) & =\left(\int_{2}^{t}\left(\frac{1}{a(s)}\right)^{1 / \gamma} \Delta s\right)^{-1} \\
& =\left(\int_{2}^{t} s^{-2 / 5} \Delta s\right)^{-1}=\frac{2^{3 / 5}-1}{t^{3 / 5}-2^{3 / 5}} ;
\end{aligned}
$$


then, as $t \rightarrow \infty$,

$$
\begin{gathered}
\int_{2}^{t}\left[L \delta(s) q(s)-\eta^{\gamma}(s)\left(\delta^{\Delta}(s)-\delta(s) \frac{p(s)}{a(s)}\right)\right] \Delta s \\
\geq \int_{2}^{t}\left[\frac{1}{s}-\left(\frac{2^{3 / 5}-1}{s^{3 / 5}-2^{3 / 5}}\right)^{5 / 3}\left(s^{3 / 5}\right)^{\Delta}\right] \Delta s \longrightarrow \infty
\end{gathered}
$$

so (10) is satisfied as well. Altogether, by Theorem 3, (30) is oscillatory.

\section{Conflict of Interests}

The authors declare that there is no conflict of interests regarding the publication of this paper.

\section{Acknowledgments}

The authors sincerely thank the reviewers and editors for their valuable suggestions and useful comments that have led to the present improved version of the original paper. This work was supported by the Natural Science Foundation of Shandong Province of China under Grant no. ZR2013AM003 and the Development Program in Science and Technology of Shandong Province of China under Grant no. 2010GWZ20401.

\section{References}

[1] S. R. Grace, M. Bohner, and R. P. Agarwal, "On the oscillation of second-order half-linear dynamic equations," Journal of Difference Equations and Applications, vol. 15, no. 5, pp. 451-460, 2009.

[2] R. P. Agarwal, M. Bohner, and S. H. Saker, "Oscillation of second order delay dynamic equations," Canadian Applied Mathematics Quarterly, vol. 13, pp. 1-18, 2005.

[3] Y. Şahiner, "Oscillation of second-order delay differential equations on time scales," Nonlinear Analysis: Theory, Methods and Applications, vol. 63, no. 5-7, pp. e1073-e1080, 2005.

[4] L. Erbe, A. Peterson, and S. H. Saker, "Oscillation criteria for second-order nonlinear delay dynamic equations," Journal of Mathematical Analysis and Applications, vol. 333, no. 1, pp. 505522, 2007.

[5] S. Sun, Z. Han, and C. Zhang, "Oscillation of second-order delay dynamic equations on time scales," Journal of Applied Mathematics and Computing, vol. 30, no. 1-2, pp. 459-468, 2009.

[6] L. Erbe, T. S. Hassan, and A. Peterson, "Oscillation criteria for nonlinear functional neutral dynamic equations on time scales," Journal of Difference Equations and Applications, vol. 15, no. 1112, pp. 1097-1116, 2009.

[7] S. R. Grace, R. P. Agarwal, B. Kaymakçalan, and W. Sae-Jie, "Oscillation theorems for second order nonlinear dynamic equations," Journal of Applied Mathematics and Computing, vol. 32, no. 1, pp. 205-218, 2010.

[8] Q. Zhang and L. Gao, "Oscillation of second-order nonlinear delay dynamic equations with damping on time scales," Journal of Applied Mathematics and Computing, vol. 37, no. 1-2, pp. 145158, 2011.

[9] M. Bohner and A. Peterson, Dynamic Equations on Time Scales, An Introduction with Applications, Birkhäuser, Boston, Mass, USA, 2001.
[10] R. P. Agarwal, M. Bohner, S. R. Grace, and D. O’Regan, Discrete Oscillation Theory, Hindawi, New York, NY, USA, 2005.

[11] M. Bohner, "Some oscillation criteria for first order delay dynamic equations," Far East Journal of Applied Mathematics, vol. 18, no. 3, pp. 289-304, 2005. 


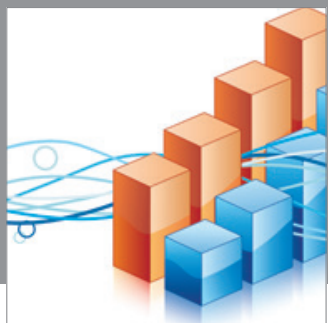

Advances in

Operations Research

mansans

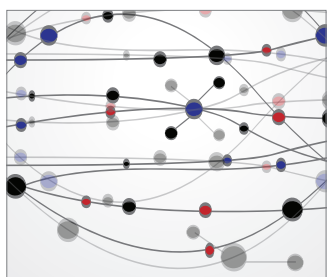

The Scientific World Journal
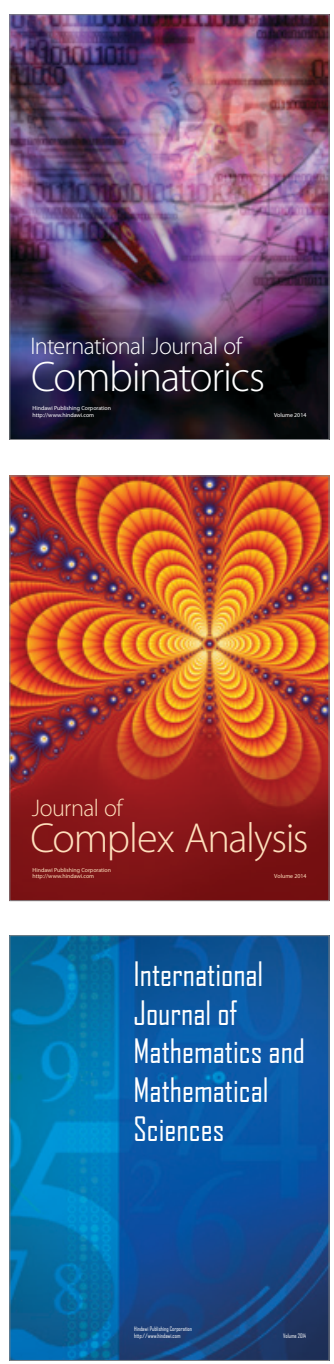
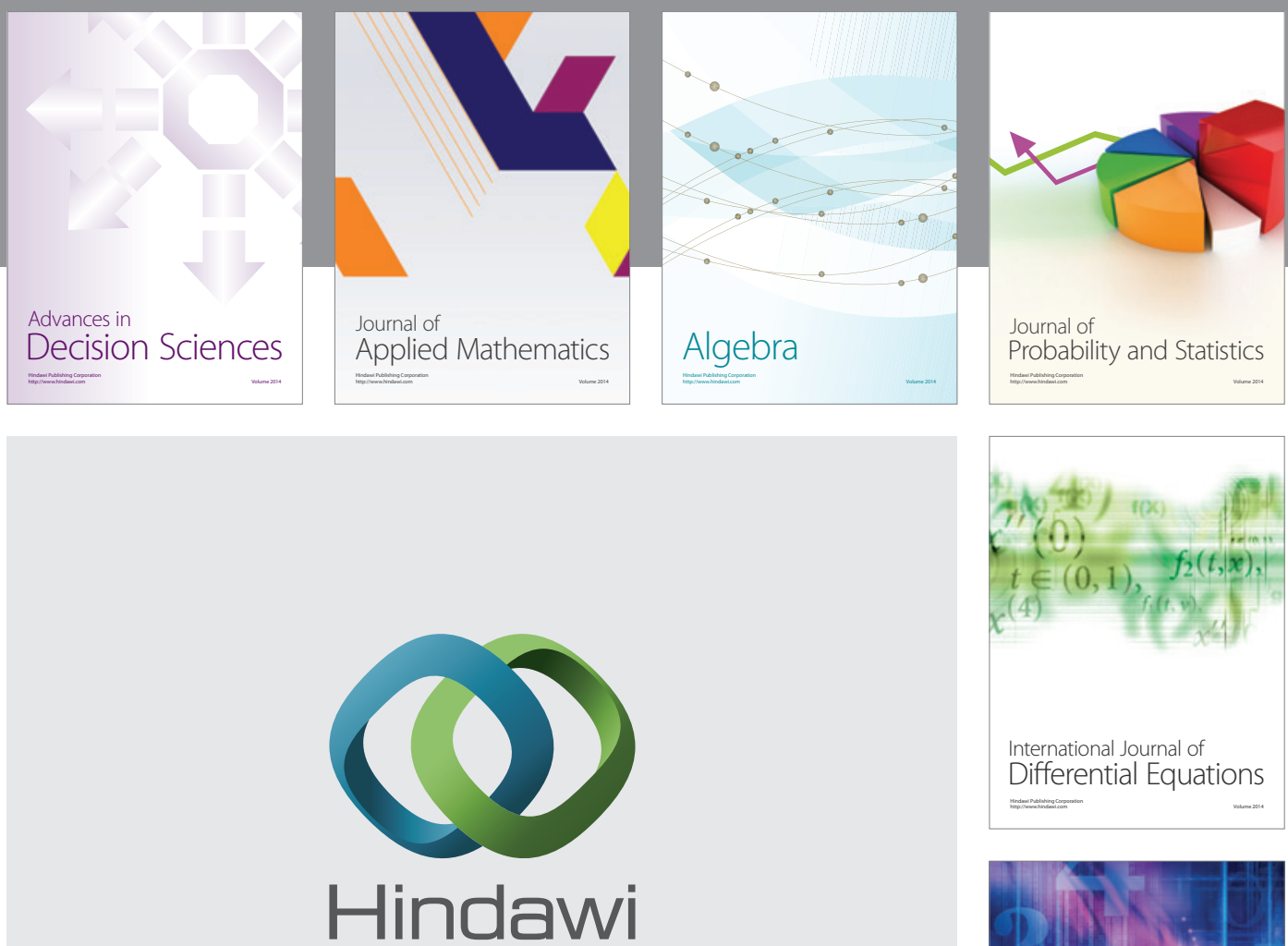

Submit your manuscripts at http://www.hindawi.com
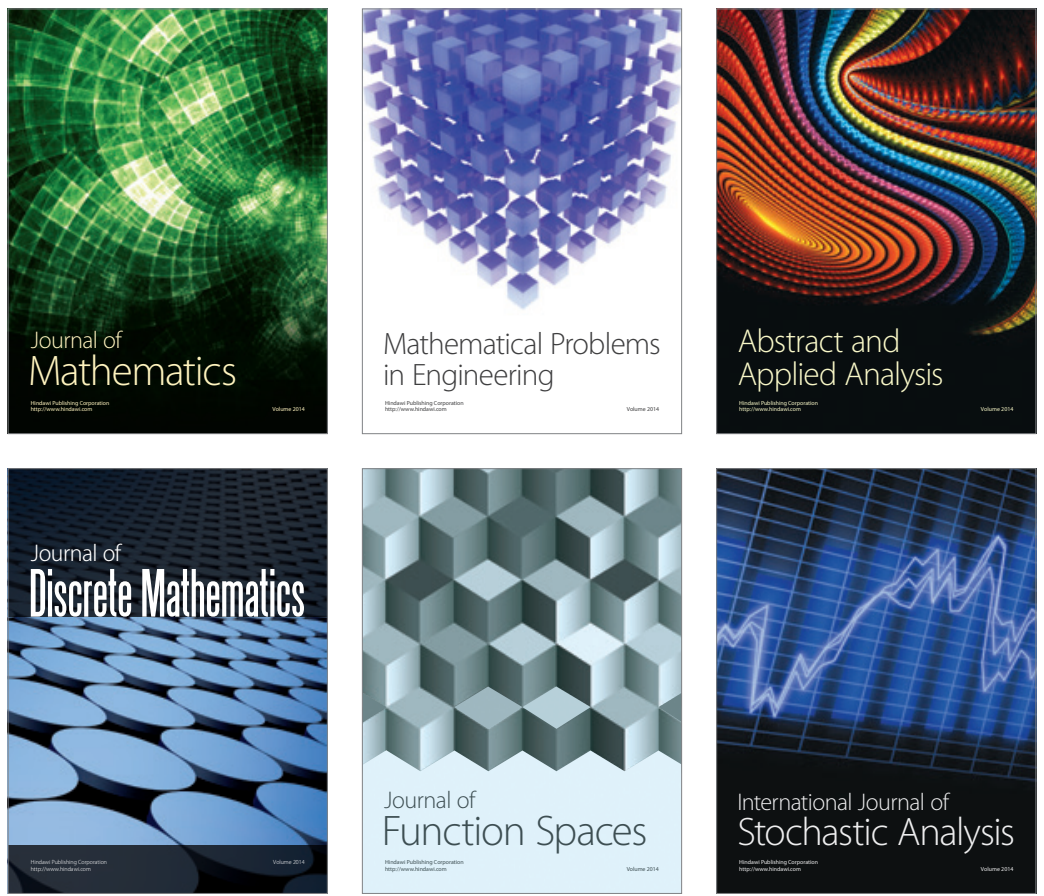

Journal of

Function Spaces

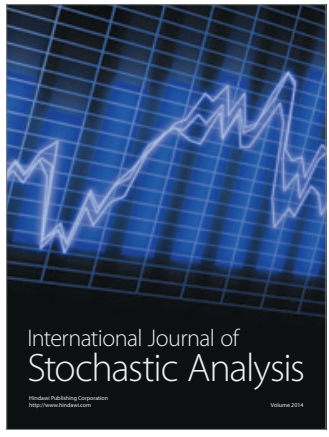

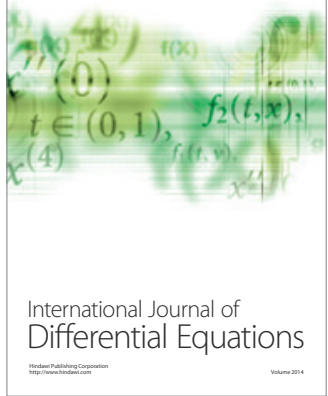
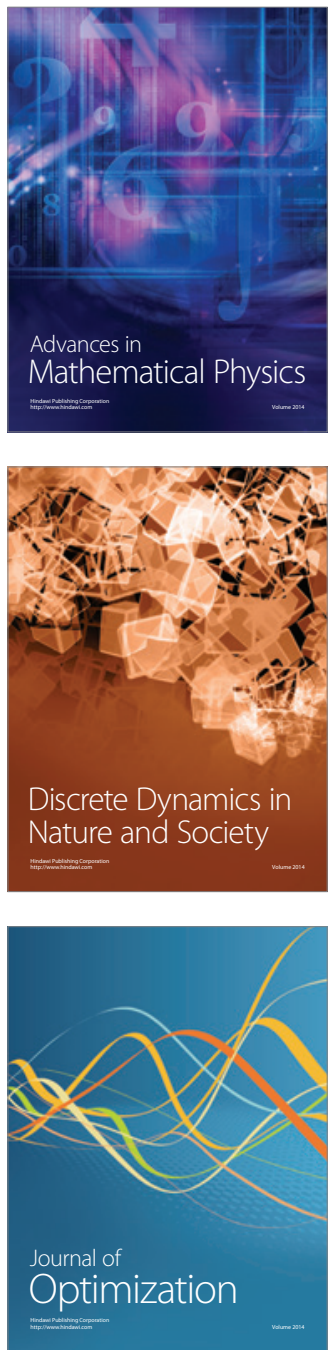